\title{
Irreversible Enzyme-Shuttle Immunoassay
}

\author{
Se-Hwan Paek \\ University of Michigan, Reproductive Sciences Program and Bioengineering \\ Program, 300 N. Ingalls, Ann Arbor, Michigan 48109 \\ Willfried Schramm* \\ University of Michigan, Reproductive Sciences Program; and BioQuant, Inc., \\ 1919 Green Rd., Ann Arbor, Michigan 48105
}

Received May 3, 1991/Accepted October 11, 1991

The concept of a competitive enzyme immunoassay that utilizes simultaneously the bound and the free analyteenzyme conjugate (heterobifunctional conjugate) for signal generation in response to varying analyte concentrations in samples has been investigated. Two antigenic sites of the heterobifunctional conjugate are used in the assay for binding to immunoglobulins: the analyte derivative binds to an immobilized antibody, $A b_{1}$, and the enzyme component binds to a spatially separated immobilized antibody, $A b_{2}$. The analytical system is set up such that in the absence of analyte, the conjugate is predominantly bound in the compartment that contains $A b_{1}$. With increasing concentration of native analyte in samples, an increasing amount of conjugate migrates to the second compartment that contains $A b_{2}$. The enzyme bound in each compartment is used for signal generation. Mathematical models have been developed to determine the optimal conditions and to predict the performance of such dual-antibody systems. The theoretical predictions are supported by experimental results. The dual-antibody system has been compared with a conventional competitive enzyme immunoassay using the same reagents.

Key words: immunoassay - mathematical modeling $\cdot$ analyte monitoring

\section{INTRODUCTION}

Competitive immunoassay is the most commonly used method for the quantitative determination of small analyte in samples. ${ }^{7,11,19}$ In these assays, an excess of labeled analyte (e.g., radiolabeled derivative or analyte-enzyme conjugate, the tracer $)^{16}$ competes with native analyte from the sample for limited binding sites at the antibody. One of the characteristics of this type of assay is that free antigen (i.e., not bound to the antibody) needs to be separated from the antibody-bound antigens to obtain a meaningful reading for quantitative analysis. As a consequence, excess labeled tracer is discarded and not available for signal generation.

We have investigated an analytical system that utilized simultaneously the bound as well as the excess, i.e., the free, tracer for signal generation in competitive enzyme immunoassays. ${ }^{15}$ Two antibodies are present during the formation of the antigen-antibody complex: one antibody to the native and the labeled analyte, and

\footnotetext{
* To whom all correspondence should be addressed.
}

another antibody to the enzyme that constitutes the tracer (signal generator) in the analyte-enzyme conjugate. In response to different analyte concentrations, the analyte-enzyme conjugate can shuttle between the two antibodies that are immobilized on separate solid matrices.

In this article, we describe the theoretical basis for this dual-antibody system and compare mathematical models with experimentally obtained results. In particular, the signal yields and the sensitivities of the traditional enzyme immunoassay and the dual-antibody method are compared.

We will report elsewhere on the application of the dual-antibody assay with a heterobifunctional binder as signal generator in a disposable strip test based on immunochromatography. The concept can also be applied to the construction of sensors that are suitable for continuous monitoring of analytes in a medium stream. ${ }^{15}$

\section{DEFINITION OF TERMS}

\section{Experimental Model}

Two different antibodies as binding proteins are immobilized on spatially separated solid surfaces [Fig. 1(A)]. One antibody $\left(A b_{1}\right)$ recognizes the analyte to be detected. The other antibody $\left(A b_{2}\right)$ binds specifically to the signal generator, e.g., an enzyme. The heterobifunctional signal generator, consisting of one analyte molecule bound covalently to one enzyme, can bind to either antibody. However, conditions can be selected such that the heterobifunctional conjugate $(\mathrm{Co})$ binds predominantly to $A b_{1}$ at the ground state, i.e., in the absence of external analyte [Fig. 1(B)]. This can be achieved by selecting the appropriate ratios of either the binding constants or the concentrations of the two antibodies to the respective antigens. External analyte from a sample competes with the conjugate $\mathrm{Co}$ for binding sites on $A b_{1}$, and eventually the conjugate dissociates and is then captured by $A b_{2}$ [Fig. 1(C)]. As a result, the presence of analyte causes a shuttling of the signal generator (analyte-enzyme conjugate) from the area occupied by antibody $A b_{1}$ to the area containing antibody $A b_{2}$. 


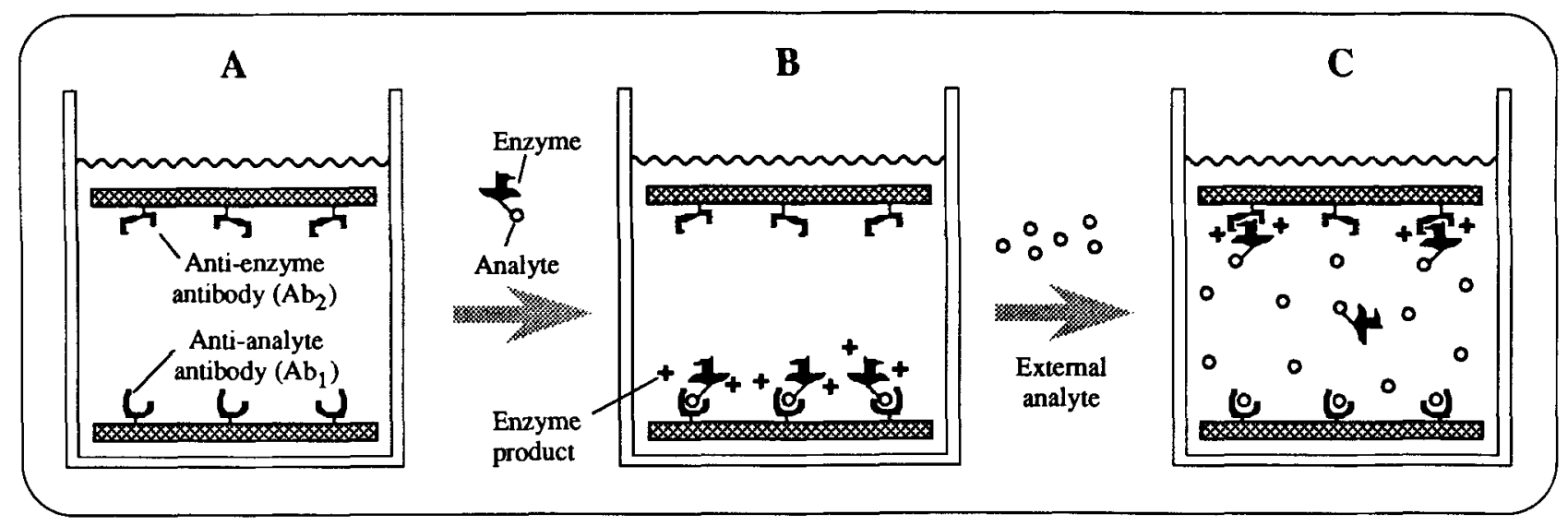

Figure 1. Experimental model of the enzyme-shuttle immunoassay. The model consists of two binding proteins, i.e., antibodies to the analyte and to the enzyme (A). The heterobifunctional conjugate that binds to either antibody is added and binds predominantly to analyte antibody (B) as a result of controlled equilibrium conditions (see text). Upon adding of external analyte, competitive binding between two species (native and enzyme-conjugated analyte) results in the dissociation of the conjugate from the analyte antibody and the conjugate eventually binds to the enzyme antibody $(\mathrm{C})$.

\section{Mathematical Model}

We developed an equilibrium model of antigen-antibody binding to describe the theoretical basis of the analytical system shown in Figure 1. Because the concentrations of conjugate, i.e., signal generator, on the two antibodies provides a differential signal that reflects the native analyte concentration, we will first study mathematical models to determine the concentrations of analyte-enzyme conjugate bound to $A b_{1}$ and $A b_{2}$.

We used the following five equations for the modeling:

1. two equations for the equilibrium binding reactions of the conjugate with $A b_{1}$ and $A b_{2}$,

2. one equation for the equilibrium reaction of the external analyte with $A b_{1}$, and

3. two equations from the law of mass action for each antibody interacting with its antigen, i.e., total antibody $=$ unoccupied antibody + antigen-antibody complex.

The five equations were used to express the concentrations of bound conjugate to each antibody, $\left[A b_{1}: C o\right]$ and $\left[\mathrm{Ab}_{2}: \mathrm{Co}\right]$, by means of unbound conjugate, $\mathrm{Co}$, and native analyte $\mathbf{P}$ (analyte from the sample).

$$
\begin{gathered}
{\left[A b_{1}: C o\right]=\frac{K_{a 1}\left[A b_{t 1}\right][C o]}{1+K_{a 1}[C o]+K_{a p}[P]}} \\
{\left[A b_{2}: C o\right]=\frac{K_{a 2}\left[A b_{t 2}\right][C o]}{1+K_{a 2}[C o]}}
\end{gathered}
$$

Equations (1) and (2) allow us to obtain the concentration of unbound conjugate $\mathrm{Co}$ as a function of the analyte, so that the concentrations of bound conjugate, $\left[A b_{1}: C o\right]$ and $\left[A b_{2}: C o\right]$, can be expressed by the only independent variable $[P]$. If $\left[\mathrm{Co}_{t}\right]$ is the total concentration of conjugate, $\left[\mathrm{Co}_{t}\right]=[\mathrm{Co}]+\left[\mathrm{Ab}_{1}: \mathrm{Co}\right]+\left[\mathrm{Ab}_{2}: \mathrm{Co}\right]$. Substituting $\left[A b_{1}: C o\right]$ and $\left[A b_{2}: C o\right]$ in this equation with (1) and (2), the following expression (3) is obtained.

$$
\left[C o_{l}\right]=[C o]+\frac{K_{a 1}\left[A b_{t 1}\right][\mathrm{Co}]}{1+K_{a 1}[\mathrm{Co}]+K_{a p}[P]}+\frac{K_{a 2}\left[A b_{t 2}\right][\mathrm{Co}]}{1+K_{a 2}[\mathrm{Co}]}
$$

Equation (3) is nonlinear and, therefore, analytically difficult to solve for $[\mathrm{Co}]$ as a function of the independent variable $[P]$. However, certain boundary conditions, e.g., the initial and the final states of the immunoassay system as defined later, allow us to simplify the mathematical expressions.

\section{Differential Signal}

Two antibodies are involved in binding the analyteenzyme conjugate (the signal generator), and the amount of conjugate bound to both antibodies, $A b_{1}$ and $A b_{2}$, is proportional to the analyte concentration. Therefore, the conjugate bound to both antibodies can be used to monitor a differential signal $(D S)$ that is defined by $\left(\left[A b_{1}: C o\right]-\left[A b_{2}: C o\right]\right)$. We want to optimize the equilibrium such that in the absence of external analyte (initial state, $[P]=0$ ) the majority of conjugate molecules is bound at $A b_{1}$. At a given $K_{a 1},\left[A b_{t 1}\right], K_{a 2}$, and $\left[A b_{12}\right]$, we can calculate the maximal differential signal for the unbound conjugate concentration at the initial state $[C o]$, from $d(D S) / d[C o]=0$ by substituting $D S$ with eqs. (1) and (2).

$$
[C o]_{i}=\frac{1-\alpha / K}{K K_{a 2}(\alpha-1 / K)}
$$

where $K=\left(K_{a 1} / K_{a 2}\right)^{1 / 2}$ and $\alpha=\left(\left[A b_{12}\right] /\left[A b_{11}\right]\right)^{1 / 2}$. The optimal $[\mathrm{Co}]_{i}$ such determined, we can now calculate the differential signal at the initial state $\left(D S_{i}\right)$.

$$
D S_{i}=\left[A b_{1}: C o\right]_{i}-\left[A b_{2}: C o\right]_{i}=\frac{\left[A b_{t 1}\right](1-\alpha / K)^{2}}{\left(1-1 / K^{2}\right)}
$$

In addition, $[\mathrm{Co}]_{i}$ permits us to calculate the total concentration of conjugate, $\left[\mathrm{Co}_{t}\right]$ (i.e., bound to both anti- 
bodies + unbound), from eq. (3) by setting $[P]=0$ [see eq. (6.4)].

Native analyte will compete with the conjugate for the binding sites of the analyte antibody, $A b_{1}$. An increase in the concentration of the analyte causes proportionally increased dissociation of the conjugate bound to $A b_{1}$. We define the final state when no conjugate is bound to the analyte antibody in the presence of a high concentration (excess) of the analyte [Fig. 1(C)]. The maximal amount of conjugate that can bind at the final state to the enzyme antibody depends on the concentration of total conjugate, the affinity constant of $A b_{2}$, and the concentration of this antibody.

At this state, eq. (3) for $\left[\mathrm{Co}_{t}\right]$ is simplified: the second term of the right-hand side is eliminated because none of the conjugate is bound to $A b_{1}$. This equation can now be solved for the unknown concentration of unbound conjugate $[\mathrm{Co}]$ at the final state $\left([\mathrm{Co}]_{f}\right)$. From the calculated positive root of $[\mathrm{Co}]_{f}$ [see eq. (6.1)], the differential signal, $D S_{f}$, at the final state is obtained:

$$
D S_{f}=-\left[A b_{2}: C o\right]_{f}=-\frac{K_{a 2}\left[A b_{t 2}\right][C o]_{f}}{\left(1+K_{a 2}[C o]_{f}\right)}
$$

where

$$
\begin{gathered}
{[C o]_{f}=\frac{A_{2}-A_{1}}{2 K_{a 2}}} \\
A_{1}=1+\left(\frac{\alpha}{K}\right)^{2} K_{a 1}\left[A b_{t 1}\right]-K_{a 2}\left[C o_{t}\right] \\
A_{2}=\sqrt{A_{1}^{2}+4 K_{a 2}\left[C o_{t}\right]} \\
{\left[C o_{t}\right]=\frac{1}{K K_{a 2}} \frac{(1-\alpha / K)}{(\alpha-1 / K)}+\frac{\left[A b_{t 1}\right]\left(1-\alpha^{2} / K^{2}\right)}{\left(1-1 / K^{2}\right)}}
\end{gathered}
$$

We have now determined the optimal conjugate concentration and the differential signals at this concentration for the two boundary conditions: absence and excess of native analyte. The signals for dose-response curves will give values between these extremes. This provides the basis for calculating the signal yield and the sensitivity of the assay.

\section{Signal Yield}

For designing optimal immunoassays, it is desirable to obtain a maximal signal yield in response to analyte concentrations. The signal yield is defined as the ratio of the total change of signal in dose-response curves over a given input signal. Because the quantity of signal is difficult to express mathematically, we use the amount of conjugate contributing to the signal generation for calculations. With two antibodies binding the analyteenzyme conjugate, the signal yield represents the sum of differential signal on the two detectors (i.e., the compartments containing $A b_{1}$ and $\left.A b_{2}\right)$ at the initial and final state divided by the total conjugate, $\left(D S_{i}-D S_{f}\right) /$ $\left[\mathrm{Co}_{t}\right]$. We will compare the signal yield of the dual- antibody system with that of a representative conventional immunoassay.

In a conventional immunoassay, the maximal signal that can be obtained is determined by the amount of the immobilized antibody $\left(A b_{1}\right)$. In the border case, all the idiotypic sites of this antibody are occupied by analyteenzyme conjugate, i.e., $\left[A b_{i 1}\right]=\left[A b_{1}\right.$ :Co $]$. However, this state is not reached in practical terms. If we express the practical signal yield, $B_{0} /\left[\mathrm{Co}_{t}\right]=\left[\mathrm{Ab_{1 }}: \mathrm{Co}\right]_{i} /\left[\mathrm{Co}_{t}\right]$, as a fraction of the maximal signal yield, we obtain $B_{0}=r\left[A b_{t 1}\right]$ where $r$ is defined as the ratio of the practical yield to the theoretical, maximal yield. The ratio $r$ depends on the concentration and binding constant of the antibody and the concentration of the conjugate. $\left[A b_{1}: C o\right]$ in eq. (1) is substituted with $B_{0}$ at $[P]=0$, and $[\mathrm{Co}]$ in this expression is then replaced by $\left(\left[\mathrm{Co}_{\mathrm{t}}\right]-\mathrm{B}_{0}\right)$ from the mass balance equation for the conjugate. Because $B_{0}$ is equal to $r\left[A b_{t 1}\right]$, the final expression can be solved for $r$ to obtain eq. (7).

$$
r=\frac{C-\sqrt{C^{2}-4 K_{a 1}\left[A b_{t 1}\right] \overline{K_{a 1}\left[C o_{t}\right]}}}{2 K_{a 1}\left[A b_{t 1}\right]}
$$

where

$$
C=1+K_{a 1}\left[C o_{t}\right]+K_{a 1}\left[A b_{t 1}\right]
$$

The ratio $r$ is a measure for reaching the maximal (theoretical) signal yield in conventional competitive immunoassays. Ideally, $r$ will approach unity; in practice, however, it will be lower. For example, in the conventional assay described below with the reagents used for these investigations, $r$ is about $0.5-0.6$ if $70 \%$ of the total tracer is bound in the absence of native antigen $\left(B_{0}\right)$.

By using eqs. (5)-(7), the ratio of the signal yields, $\left(D S_{i}-D S_{f}\right) / B_{0}$, of the model system to the conventional system is calculated as follows:

$$
\frac{\left(D S_{i}-D S_{f}\right)}{B_{0}}=\frac{1}{r}\left\{\frac{(1-\alpha / K)^{2}}{\left(1-1 / K^{2}\right)}+\frac{\alpha^{2} K_{a 2}[C o]_{f}}{\left(1+K_{a 2}[C o]_{f}\right)}\right\}
$$

The calculated ratio from eq. (8) shows which system generates a higher maximal signal under comparable conditions, i.e., the same concentration of total conjugate and the antibody to analyte. If the ratio is greater than unity, the dual-antibody system is more efficient in the utilization of the conjugate for signal generation.

\section{Sensitivity}

The sensitivity of immunoassays is often defined as the minimal concentration ${ }^{4}$ of detectable analyte, although this definition does not consider the slope ${ }^{6}$ of doseresponse curves. For practical purposes, we will refer in the following to sensitivity as the amount of analyte that is required to displace from the antibody $50 \%$ of conjugate $\left(E_{50}\right) .{ }^{1,5}$ We will now provide the mathematical basis for comparing the $\mathrm{ED}_{50}$ of the conventional and the dual-antibody assay. 
Because a conventional system involves only $A b_{1}$, the dose-response curve is obtained as the plot of the bound conjugate $\left[A b_{1}: C o\right]=B$ vs. $\left[P_{t}\right] .\left[P_{t}\right]$ is the total concentration of the unbound, $[P]$, and the bound analyte to the antibody. For convenience, $\left[P_{t}\right]$ is first expressed as a function of $[P]$ by using the law of mass action for the analyte and $A b_{1}$, and the equilibrium equations between the antigens and the antibody. $[P]$ is then calculated from eq. (1). [Co] in this equation can be substituted with $\left(\left[C O_{t}\right]-B\right)$ if the concentration of the conjugate is expressed by the law of mass action. Consequently, $\left[P_{t}\right]$ can be expressed with respect to $B$ at the given concentration and binding constant of the antibody. Then, at $B=B_{0} / 2=r\left[A b_{t 1}\right] / 2,\left[P_{t}\right]$ is equal to $\mathrm{ED}_{50}$ for the conventional system as shown in eq. (9).

$$
\begin{aligned}
& \left(E D_{50}\right)_{C o n v}= \\
& {[P]_{50}\left(1+\frac{K_{a p}\left[A b_{t 1}\right]}{K_{a 1}\left(\left[C o_{t}\right]-r\left[A b_{t 1}\right] / 2\right)+K_{a p}[P]_{50}+1}\right)}
\end{aligned}
$$

where

$$
[P]_{50}=\frac{1}{K_{a p}}\left\{\left(\frac{2}{r}-1\right)\left(K_{a 1}\left[C o_{l}\right]-\frac{r K_{a 1}\left[A b_{t 1}\right]}{2}\right)-1\right\}
$$

Because the dose-response curve of the enzyme-shuttle system is represented by a plot of the differential signal (DS $\left.=\left[A b_{1}: C o\right]-\left[A b_{2}: C o\right]\right)$ against $\left[P_{l}\right],\left[P_{l}\right]$ is equal to $\mathrm{ED}_{50}$ at $D S=\left(D S_{i}+D S_{f}\right) / 2$. Under this condition, $[\mathrm{Co}]$ is equal to $[\mathrm{Co}]_{50}$, and the $\mathrm{ED}_{50}$ for the model is

$$
\left(E D_{50}\right)_{\text {Model }}=[P]_{50}\left(1+\frac{K_{a p}\left[A b_{i 1}\right]}{K_{a 1}[C o]_{50}+K_{a p}[P]_{50}+1}\right)
$$

where

$$
\begin{aligned}
& {[P]_{50}=} \\
& \frac{1}{K_{a p}}\left\{\frac{K^{2} K_{a 2}[C o]_{50}}{\frac{D S_{50}}{\left[A b_{i 1}\right]}+\frac{\alpha^{2} K_{a 2}[C o]_{50}}{1+K_{a 2}[C o]_{50}}}-K^{2} K_{a 2}[C o]_{50}-1\right\}
\end{aligned}
$$

Because $D S$ is defined as $\left(\left[A b_{1}: C o\right]-\left[A b_{2}: C o\right]\right)$, it is a linear function of $\left[A b_{1}: C o\right]$ and $\left[A b_{2}: C o\right]$ if the doseresponse curves, $\left[A b_{1}: C o\right]$ and $\left[A b_{2}: C o\right]$ vs. $\left[P_{t}\right]$ are symmetric. Dose-response curves in immunoassays can become nonsymmetric if the concentration ratio of antibody to conjugate increases. ${ }^{9}$ This occurs, however, at a ratio that is not optimal for achieving a sensitive assay. Another source for nonsymmetric curves are heterogeneous antibody or tracer populations. ${ }^{2}$ We assume negligible deviation form symmetry for the sigmoidal dose-response curves in the dual-antibody assay because the antibody/tracer ratio needs to be optimized, and best results will be obtained with monoclonal antibodies and homogeneous analyte-enzyme conjugates. In this case, $D S$ changes in a linear fashion with $[\mathrm{Co}]$ based on the relation $[\mathrm{Co}]=\left[\mathrm{Co}_{t}\right]-\left[\mathrm{Ab}_{1}: \mathrm{Co}\right]-\left[\mathrm{Ab}_{2}: \mathrm{Co}\right]$. Therefore, at $D S_{50}=\left(D S_{i}+D S_{f}\right) / 2,[C o]_{50}=\left([C o]_{i}+\right.$ $\left.[C o]_{f}\right) / 2$. Expressions for $[C o]_{i}, D S_{i},[C o]_{f}$, and $D S_{f}$ are calculated from eqs. (4), (5), (6.1), and (6), respectively.
Consequently, the ratio of $\left(\mathrm{ED}_{50}\right)_{\text {Model }} /\left(\mathrm{ED}_{50}\right)_{\text {Conv }}$ can be calculated from eqs. (9) and (10), which represents the inverse of the sensitivity ratio, because the system with a lower $E D_{50}$ shows a higher sensitivity.

For the purpose of these calculations, we assume that $K_{a p}=K_{a 1}$ (i.e., the binding constants of the analyteenzyme conjugate and the analyte to the antibody are the same). A difference in these binding constants would have little effect for the calculations because we are comparing the relative performance of the traditional assay with the dual-antibody system.

\section{Dimensionless Variables}

To obtain quantitative values for these ratios, the binding constants of the participating antibodies and their surface densities on the solid matrix, expressed as concentrations, are required. These are incorporated into three factors that determine the signal yield ratios and the sensitivity ratios: the product between the binding constant of $A b_{1}$ and the concentration of this antibody, $K_{a 1}\left[A b_{t 1}\right]$; the ratio of the binding constants, $K_{a 1} / K_{a 2}$; and the ratio of the antibody concentrations, $\left[A b_{t 2}\right] /\left[A b_{t 1}\right]$.

Ratios of Signal Yield and $\mathrm{ED}_{50}=$

Function of $K_{a 1}\left[A b_{t 1}\right], K_{a 1} / K_{a 2}$, and $\left[A b_{t 2}\right] /\left[A b_{t 1}\right]$

These three dimensionless variables regulate the performance characteristics of the dual-antibody assay.

\section{MATERIALS AND METHODS}

\section{Materials}

Horseradish peroxidase (HRP, E.C. 1.11.1.7.; type VI, 300 units/mg solid, RZ:3.0), 1,5-diaminopentane (cadaverine), poly-L-lysine hydrobromide (MW 421,000 by viscosity test), and 3,3',5,5'-tetramethylbenzidine (TMB) were purchased from Sigma (St. Louis, MO). Disuccinimidyl suberate (DSS), CNBr-activated Sepharose $4 \mathrm{~B}$, Bio Gel P-30, and polypropylene monofilament cloth (113 mesh/inch) were purchased from Pierce (Rockford, IL), Pharmacia Fine Chemicals, Inc. (Piscataway, NJ), Bio-Rad (Richmond, CA), and Small Parts Inc. (Miami, FL), respectively. Monoclonal antibody specific to HRP $\left(A b_{2}\right.$, E47) was obtained from Zymed Laboratories Inc. (San Francisco, CA). The following monoclonal antibodies were produced in this laboratory ${ }^{15}$ : two antibodies to progesterone, one with a high affinity constant $\left(A b_{1}, \mathrm{BQ} .1\right)$ and the other with a low affinity constant (4C10); one antibody to HRP ( $\left.A b_{2}, 9 \mathrm{G} 9\right)$; and one antibody to urease for estimating nonspecific binding $(N S-A b)$. Progesterone derivatives were synthesized and characterized by high-performance liquid chromatography (HPLC): progesterone-11 $\alpha$ - $N$-hydroxy succinimide (P-NHS); and progesterone- $11 \alpha$-hemisuccinyl-1,5-diaminopentane (P-CAD) by reacting P-NHS with cadaverine. 


\section{Immobilization of Antibodies on Disks}

Polypropylene monofilament mesh disks (6-mm diam.) were prepared with the following antibodies immobilized on separate disks: $A b_{1}(\mathrm{BQ} .1), A b_{2}$ (9G9 and E47), and $N S-A b$. The immunoglobulins were immobilized by a modified procedure of the sodium periodate method. ${ }^{10,13}$ The disks were cleaned in nitric acid, polyL-lysine was coated on the surface, the lysine was reacted with antibody activated by $\mathrm{NaIO}_{4}$, and the resulting imide bonds were reduced with $\mathrm{NaCNBH}_{3}$ as described elsewhere. ${ }^{15}$

\section{Preparation of a Defined Progesterone-HRP Conjugate}

The progesterone derivative, P-CAD, was chemically reacted with the enzyme, HRP, via DSS as a cross-linking reagent. The conjugates were synthesized by: (a) reacting P-CAD with two-molar excess of cross-linking reagent; (b) after incubation for $30 \mathrm{~min}$ on a shaker, adding this mixture to enzyme with 20 -mol excess of P-CAD; and (c) incubation for $2 \mathrm{~h}$ on a shaker. The reaction mixture of progesterone-HRP conjugate was dialyzed in $0.01 \mathrm{~mol} / \mathrm{L}$ phosphate buffer, $\mathrm{pH} 7.0$, containing $0.14 \mathrm{~mol} / \mathrm{L} \mathrm{NaCl}$ and $0.02 \%$ (wt/vol) thimerosal (PBS), and then partially purified by exclusion chromatography on Bio Gel P-30.

The conjugation mixture was finally purified on an immuno-affinity column $(1.1 \mathrm{~cm} \times 37 \mathrm{~cm}, 20-\mathrm{mL}$ bed volume) with the IgG to progesterone, $4 \mathrm{C} 10$, immobilized on CNBr-activated Sepharose $4 \mathrm{~B}$ gel. The purified conjugate with one progesterone molecule bound to one HRP molecule (P-HRP) was selected, diluted with the same volume of PBS with $0.1 \%$ (wt/vol) gelatin (gelPBS), and stored at $-4^{\circ} \mathrm{C}$. The concentration of P-HRP in solution was determined by comparing its activity in a solid-phase assay with standard concentration of free HRP. The enzymatic activity of the conjugate was preserved without loss for more than 1 year.

\section{Measurement of HRP Activity}

To measure the activity of the HRP conjugate bound to antibodies on disks, unbound conjugate was removed from the disks by washing with deionized water, the disks were placed separately in nontreated microwells, and $200 \mu \mathrm{L}$ of a substrate solution for HRP was added. The substrate solution contained: $10 \mu \mathrm{L}$ of $3 \%$ (vol/vol) $\mathrm{H}_{2} \mathrm{O}_{2}$ in water; $100 \mu \mathrm{L}$ of $10 \mathrm{mg} / \mathrm{mL}$ TMB in dimethyl sulfoxide; and $10 \mathrm{~mL}$ of $0.05 \mathrm{~mol} / \mathrm{L}$ acetate buffer, pH 5.1. After developing color from the chromogen $\mathrm{TMB}^{3}$ at indicated times, $50 \mu \mathrm{L}$ of $0.5 \mathrm{~mol} / \mathrm{L} \mathrm{H}_{2} \mathrm{SO}_{4}$ was added. The disks were removed from the solution, and the absorbance was measured at $450 \mathrm{~nm}$.

\section{Scatchard Analysis}

Scatchard analysis ${ }^{14}$ was performed to determine both the concentrations of the antibodies immobilized on the disks and the binding constants between the conjugate P-HRP and the specific antibodies. ${ }^{15}$ Disks bearing immobilized antibody were placed in microwells, and known concentrations of the conjugate were added. The buffer used for conjugate was gel-PBS. After $5 \mathrm{~h}$ incubation, the enzymatic activity of bound conjugate was measured as described earlier, and the concentration of the bound conjugate was determined by means of a standard curve of a known amount of HRP. The nonspecific binding of the conjugate was measured from wells with NS-Ab immobilized, and subtracted from the total binding. All experiments were carried out in duplicate, and the means were used for Scatchard plots.

\section{Equilibrium Binding Curves}

Equilibrium binding curves for the complexes between P-HRP and the antibodies, $A b_{1}$ and $A b_{2}$, at the initial (without analyte) and final (with excess analyte) states were obtained to determine an optimal concentration of the conjugate. To this end, different concentrations of the conjugate were added to $A b_{1}$ immobilized on the disks as described earlier. To establish equilibrium at the final state, a standard solution of progesterone ( $20 \mu \mathrm{L}$ per well of a stock solution of $100 \mathrm{ng} / \mathrm{mL}$ ) was added. The total volume was $40 \mu \mathrm{L}$ per well for all experiments. The wells were wrapped tightly in plastic film to avoid evaporation and then incubated for $15 \mathrm{~h}$ in a box maintained at $100 \%$ humidity. After this time, the disks were washed, and the concentrations of the conjugate bound to the disks were determined as described earlier.

\section{Dose-Response Curves}

The performance of the dual-antibody method was evaluated with dose-response curves by comparison with a conventional enzyme immunoassay as the control system with one antibody on the disk. Appropriately selected disks with the immobilized antibodies were placed in microwells, and P-HRP and progesterone standards were added to obtain a total volume of $40 \mu \mathrm{L} .^{15}$ At equilibrium, the concentrations of the conjugate specifically bound to each antibody were determined as described earlier. The dose-response curves were obtained by plotting the concentration of the bound conjugate against the concentration of progesterone. For the dual-antibody system, dose-response curves for each antibody were transformed to obtain a single curve (see later).

\section{RESULTS AND DISCUSSION}

The enzyme-shuttle immunoassay utilizes two basic components: a heterobifunctional conjugate and two binding proteins immobilized on separate solid matrices. In this study, the two binding proteins were monoclonal antibodies specific to the analyte (the steroid hormone progesterone, $A b_{1}$ ), and to the enzyme (horseradish 
peroxidase, $A b_{2}$ ) as the signal generator. The two antibodies were immobilized on spatially separated polypropylene monofilament mesh disks. The disks were selected among solid matrices with different geometries (e.g., polyacrylamide rod/polystyrene tube, two glass rods) to increase the concentration of the antibodies based on unit volume. The heterobifunctional conjugate consisted of an enzyme molecule (HRP) to which one analyte molecule (progesterone) was chemically bound via a linker molecule. The concept for this enzymeshuttle immunoassay is described in Figure 1.

In comparing the dual-antibody system with a conventional competitive enzyme immunoassay, we have put forward two hypotheses:

1. The signal yield from the analyte-enzyme conjugate is higher in the dual-antibody system if $K_{a 1}>K_{a 2}$.

2. The sensitivity in the dual-antibody system is equal or higher than in a conventional assay.

The hypotheses will be investigated in the following.

\section{Performance Prediction}

The model system of the enzyme-shuttle assay was mathematically described for obtaining the signal yield and the sensitivity $\left(E D_{50}\right)$. For the evaluation of this analytical system, a competitive assay was selected as a control, and the ratios of the signal yields (eq. (8)] and $\mathrm{ED}_{50}$ [eq. (10) divided by eq. (9)] of these two systems were derived. The two expressions are determined by three major factors [eq. (11)]. The effects of these three factors are graphically represented in Figures $2-5$ by maintaining constant one factor and expressing the ratio of the signal yields or $E D_{50}$ as a function of two others. For these analyses, the same amount of immobilized antibody $A b_{1}$ and analyte-enzyme conjugate was used for the conventional assay and the corresponding dual-antibody system.

\section{Signal Yield}

We defined the signal yield as the ratio of the amount of conjugate contributing to the signal generation over

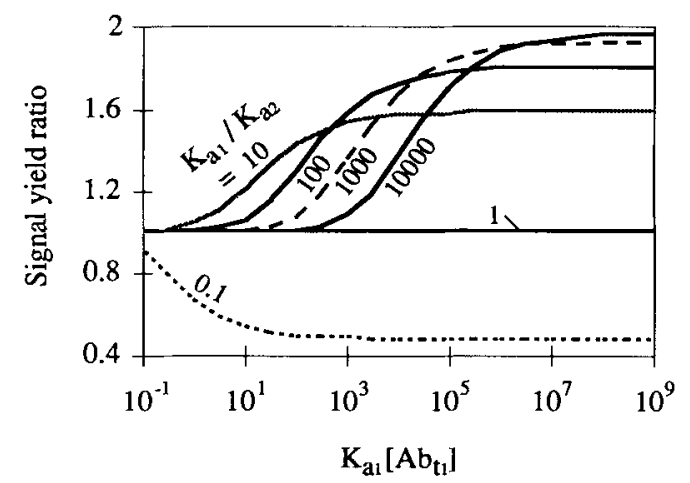

Figure 2. The signal yield ratios (i.e., dual-antibody system/ conventional assay) according to eq. (8) as a function of $K_{a 1} / K_{a 2}$ and $K_{a 1}\left[A b_{i 1}\right]$ at $\left[A b_{t 1}\right]=\left[A b_{t 2}\right]$.

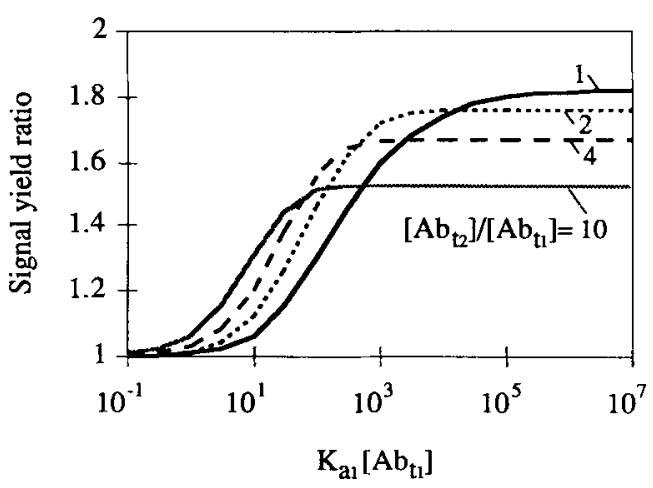

Figure 3. The signal yield ratios (i.e., dual-antibody system/ conventional assay) according to eq. (8) as a function of $\left[A b_{i 2}\right] /\left[A b_{t 1}\right]$ and $K_{a 1}\left[A b_{11}\right]$ at $K_{a 1}=100 K_{a 2}$.

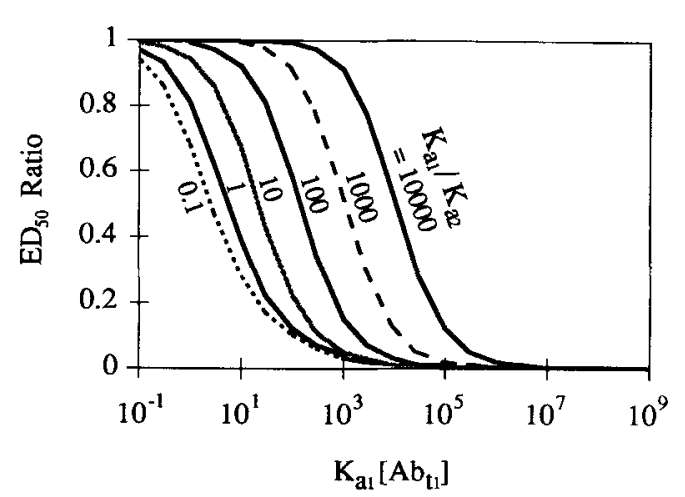

Figure 4. The $\mathrm{ED}_{50}$ ratio (i.e., dual-antibody system/conventional assay) derived from eq. (10) divided by eq. (9) as a function of $K_{a 1} /$ $K_{a 2}, K_{a 1}\left[A b_{t 1}\right]$ at $\left[A b_{t 1}\right]=\left[A b_{t 2}\right]$.

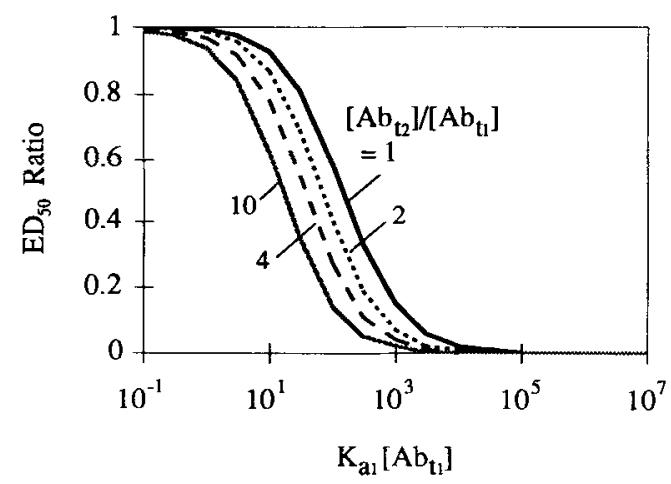

Figure 5. The $\mathrm{ED}_{50}$ ratio (i.e., dual-antibody system/conventional assay) as a function of $\left[A b_{t 2}\right] /\left[A b_{t 1}\right]$ and $K_{a 1}\left[A b_{t 1}\right]$ at $K_{a 1}=100 K_{a 2}$.

the total concentration (i.e., conjugate bound to antibody over total conjugate). In the following, we analyze the effect of each of the three factors as mentioned in eq. (11) on the signal yield in the enzyme-shuttle system and a conventional assay. The signal yield of the dualantibody method is normalized to that of the conventional assay.

1. Constant $\left[A b_{t 2}\right] /\left[A b_{t 1}\right]$ and $K_{a 1}\left[A b_{t 1}\right]$; variable $K_{a 1} / K_{a 2}$ (Fig. 2: select a given $K_{a 1}\left[A b_{t 1}\right]$ and follow the $y$ axis).

The ratio of the signal yields for the dual-antibody system and a conventional assay was plotted against two 
factors, $K_{a 1} / K_{a 2}$ and $K_{a 1}\left[A b_{t 1}\right]$ [eq. (8), Fig. 2]. The two antibody concentrations were kept equal $(\alpha=1)$. To test the effect of the binding constant of enzyme antibody $\left(A b_{2}\right), K_{a 1}$ is constant, and $K_{a 2}$ is variable to obtain the following ratios:

A. $K_{a 1} / K_{a 2}>1$. The signal yield ratio is always greater than or equal to unity. Following the $y$ axis at a given $K_{a 1}\left[A b_{t 1}\right]$, the values of the signal yield ratios for different $K_{a 1} / K_{a 2}$ depend on the selected value for $K_{a 1}\left[A b_{t 1}\right]$ because the sigmoidal curves cross over.

B. $K_{a 1} / K_{a 2}=1$. The signal yields from both systems are identical.

C. $K_{a 1} / K_{a 2}<1$. The signal yield of the dual-antibody system is lower than that of a conventional assay.

These results show that a signal yield higher than in a conventional assay can be obtained with the dualantibody system as long as a precondition applies: excess binding of conjugate to $A b_{1}$ at the initial state.

2. Constant $\left[A b_{t 2}\right] /\left[A b_{t 1}\right]$ and $K_{a 1} / K_{a 2}$; variable $K_{a 1}\left[A b_{t 1}\right]$ (Fig. 2: select a given $K_{a 1} / K_{a 2}$ and follow the $x$ axis).

A. $K_{a 1} / K_{a 2}>1$. The signal yield ratio is directly proportional to [ $\left.A b_{i 1}\right]$. At a high value of $K_{a 1} / K_{a 2}$, the signal yield ratio reaches asymptotically two.

B. $K_{a 1} / K_{a 2}=1$. The signal yields from the both systems are identical and do not change with increasing $K_{a 1}\left[A b_{t 1}\right]$.

C. $K_{a 1} / K_{a 2}<1$. The signal yield ratio $<1$ and decreases asymptotically with increasing $K_{a 1}\left[A b_{t 1}\right]$.

3. Constant $K_{a 1} / K_{a 2}$; variable $\left[A b_{t 2}\right] /\left[A b_{t 1}\right]$ or $K_{a 1}\left[A b_{t 1}\right]$.

In Figure 3 , the signal yield ratio is plotted as a function of $\left[A b_{t 2}\right] /\left[A b_{t 1}\right]$ and $K_{a 1}\left[A b_{t 1}\right]$ by keeping $K$ constant at a value of 10 at which $K_{a 1}$ is 100 times larger than $K_{a 2}$ because $K=\left(K_{a 1} / K_{a 2}\right)^{1 / 2}$. The concentrations of the antibodies are selected to obtain the values for $\left[A b_{t 2}\right] /$ $\left[A b_{11}\right]$ of $1,2,4$, and 10 . The signal yield of the dualantibody system increases at a given $\left[A b_{t 2}\right] /\left[A b_{t 1}\right]$ with increasing $K_{a 1}\left[A b_{t 1}\right]$ on the $x$ axis. For selecting an optimal ratio of $\left[A b_{t 2}\right] /\left[A b_{t 1}\right]$, it depends at what $K_{a 1}\left[A b_{t 1}\right]$ we measure because the sigmoidal curves cross over.

From the analyses of Figures 2 and 3, we confirm the first hypothesis and conclude:

1. The signal yield in the dual-antibody system is higher than that in a conventional assay if the concentration of conjugate bound to $A b_{1}$ is higher than that to $A b_{2}$ in the absence of analyte.

2. The selection of appropriate binding constants for the dual-antibody system is more critical for higher signal yield than the variation of the concentrations of these antibodies (Fig. 2 vs. Fig. 3).

3. Above a critical value $\left(K_{a 1} / K_{a 2} \geq 1\right.$, Fig. 2$)$, neither selecting the binding constants of the two antibodies to their respective antigen nor manipulating the ratios of the concentrations of the antibodies in the dual-antibody system can increase the signal yield above a factor of two compared to a conventional assay (Fig. 3).

\section{Sensitivity}

We compared dose-response curves of the dual-antibody system with those of conventional competitive enzyme immunoassays. As a criterion for these comparisons, we selected the concentration of analyte that inhibited binding of tracer by $50 \%$ at the antibody that recognizes the analyte $\left(\mathrm{ED}_{50}\right)$. In the following, we will analyze the effects of the three variables described in eq. (11) on the ratio of the $\mathrm{ED}_{50}$ from the dual-antibody and the conventional assay. The slopes of the logit-log transformations of the sigmoidal dose response curves will be examined in Experimental Models.

1. Constant $\left[A b_{t 2}\right] /\left[A b_{t 1}\right]$ and $K_{a 1}\left[A b_{t 1}\right]$; variable $K_{a 1} / K_{a 2}$ (Fig. 4: select a given $K_{a 1}\left[A b_{t 1}\right]$ and follow the $y$ axis)

Assume we keep the concentrations of the immobilized antibodies in the dual-antibody system constant and their ratio at unity (i.e., $\left[A b_{t 2}\right]=\left[A b_{11}\right]$ ) but we change the ratios of the binding constants $K_{a 1} / K_{a 2}$, e.g., by increasing $K_{a 2}$ at a constant $K_{a 1}$. Moving along the $y$ axis in Figure 4 at a given $K_{a 1}\left[A b_{i 1}\right]$, the $\mathrm{ED}_{50}$ ratio of the two assay systems decreases (i.e., the dual-antibody system is more sensitive) with decreasing values for $K_{a 1} / K_{a 2}$. In practical terms, an antibody to the enzyme, $A b_{2}$, with a higher binding constant can more efficiently remove dissociated analyte-enzyme conjugate (scavenger effect), which is then also removed from competition with native analyte for binding sites on $A b_{1}$.

The advantage of higher sensitivity with the dualantibody system can be achieved without loss of the signal yield if the appropriate constants are selected. For example, the model with $K_{a 1} / K_{a 2}=1$ and $K_{a 1}\left[A b_{t 1}\right]=10$ gives an $\mathrm{ED}_{50}$ ratio of about 0.4 (Fig. 4), i.e., the dualantibody system is 2.5 times more sensitive than a conventional assay with a signal yield identical for both systems (Fig. 2). In a conventional assay, one may use less conjugate to obtain a comparable sensitivity, which, however, results in the generation of a weaker signal.

2. Constant $\left[A b_{t 2}\right] /\left[A b_{t 1}\right]$ and $K_{a 1} / K_{a 2}$; variable $K_{a 1}\left[A b_{t 1}\right]$ (Fig. 4: select a given $K_{a 1} / K_{a 2}$ and follow the $x$ axis)

For any given set of $K_{a 1} / K_{a 2}$ (Fig. 4), the $\mathrm{ED}_{50}$ ratio is inversely proportional to $K_{a 1}\left[A b_{t 1}\right]$, i.e., the dual antibody assay is more sensitive at higher concentrations of the antibody $\left[A b_{i 1}\right]$ compared to a single antibody assay. It should be noted that, for the purpose of this analysis, we set the precondition $\left[A b_{t 2}\right]=\left[A b_{t 1}\right]$. While $\left[A b_{t 2}\right]$ is not incorporated into a conventional assay, we compensate increased $\left[A b_{t 1}\right]$ in the dual-antibody assay with proportionally increased $\left[A b_{t 2}\right]$, which may account for a sensitivity ratio favoring the dual-antibody system. In the following, we will examine the effect of different 
concentrations of the two participating antibodies in the dual-antibody assay.

3. Constant $K_{a 1} / K_{a 2}$ and $K_{a 1}\left[A b_{t 1}\right]$; variable $\left[A b_{t 2}\right] /\left[A b_{t 1}\right]$

For the analysis shown in Figure 5, we have set the ratio $K_{a 1} / K_{a 2}$ at 100 . Selecting a given $K_{a 1}\left[A b_{r 1}\right]$ and moving along the $y$ axis in Figure 5, the $\mathrm{ED}_{50}$ ratio of the two assay systems decreases with increasing $\left[A b_{t 2}\right] /\left[A b_{t 1}\right]$. In practical terms, the more $A b_{2}$ (relative to $A b_{1}$ ) is used, the more sensitive is the dual-antibody assay.

Based on these results, we confirm the second hypothesis and conclude:

1. Sensitivity. An antibody with a high binding constant to the enzyme (Fig. 4) and/or used in a relatively high concentration (Fig. 5) increases the sensitivity of the dual-antibody system relative to a traditional (single-antibody) assay.

Combining the findings from the first and the second hypothesis, we resolve:

2. However, the conditions mentioned in 1 need to be reconciled with those for an optimal signal yield by controlling the ratios of the binding constants and the concentrations of the two antibodies (see Figs. 2 and 3), e.g.:

2a. Sensitivity. A precondition for higher sensitivity compared with a single-antibody assay with a signal yield no less than in the traditional assay is the use of approximately the same amount of conjugate bound (e.g., expressed in the ratio $K_{a 1} / K_{a 2}$ at $\left[A b_{t 1}\right] /\left[A b_{t 2}\right]$, Figs. 2 and 4$)$ to each antibody in the absence of native analyte (initial state).

2b. Signal yield. To the contrary, excess binding of analyte-enzyme conjugate to the analyte antibody at the initial state is the precondition to obtain a high signal yield without decreasing sensitivity.

3. Although the relative sensitivities and signal yields between the dual-antibody system and a conventional assay are proportional to the product $K_{a 1}\left[A b_{t 1}\right]$, this factor may be a limiting variable for adjusting to optimal conditions. The binding constants of antibodies usually do not exceed $10^{12} \mathrm{~L} / \mathrm{mol} .{ }^{8,18}$ Likewise, the concentration of the analyte antibody in competitive immunoassays cannot be increased infinitely because the absolute sensitivity of an assay is inversely proportional to the amount of antibody used. ${ }^{17} \mathrm{We}$ will further discuss the resulting conclusion that the binding constant effect supersedes the concentration effect in Limitations and Applications.

We have shown theoretically that, by optimizing three factors [eq. (11)], two immunoassay characteristics can be improved by the dual-antibody method if compared with a single antibody assay: the signal yield and the sensitivity. In the following, the theoretical analyses are experimentally supported.

\section{Experimental Models}

We wanted to evaluate the dual-antibody assays with dose-response curves. To this end, we had to optimize the components of the assay.

As the theoretical analysis has shown, it is favorable to operate the assay with an antibody $A b_{1}$ that provides a high value for the product $K_{a 1}\left[A b_{t 1}\right]$. Therefore, we selected an immunoglobulin to the analyte with a high binding constant (BQ. $1^{17}$ ), and we immobilized it in a maximal concentration on the surface of polypropylene disks. Based on the experimentally determined binding constant of the immobilized antibody and the surface density as described elsewhere, ${ }^{15}$ the experiments for the dual-antibody and the conventional assay were carried out at a constant value for $K_{a 1}\left[A b_{t 1}\right]=13.9$. The dual-antibody system was then optimized for the signal yield and sensitivity by varying the ratios of the binding constants, the antibody concentrations, and the amount of conjugate.

\section{Ratios and Binding Constants}

To vary the ratio of binding constants, two antibodies to the enzyme (9G9 and E47) with different binding constants were used in combination with the antibody to the analyte (BQ.1). We will describe two experimental models for the dual-antibody assay: for Model $\mathrm{A}$, we used the combination of antibodies BQ.1 $\left(A b_{1}\right)$ and E47 $\left(A b_{2}\right)$ for which the ratio of binding constants was 4.5 (Table I); for Model B, we used the antibody combination BQ.1 $\left(A b_{1}\right)$ and $9 \mathrm{G} 9\left(A b_{2}\right)$ with a ratio of binding constants about 10 times higher $\left(K_{a 1} / K_{a 2}=48\right)$ than that in Model A.

\section{Ratios of Antibody Concentrations}

To express the signal yield and the $\mathrm{ED}_{50}$ of the dualantibody assay, we have now determined two of the three factors expressed in eq. (11): $K_{a 1} / K_{a 2}$ and $K_{a 1}\left[A b_{11}\right]$. The estimation of the remaining factor, the ratio of the antibody concentrations, $\left[A b_{t 2}\right] /\left[A b_{t 1}\right]$, would require a substantial amount of experimentation if no indication as to the optimal ratio is available. Fortunately, we can estimate the approximate value for the effective ratio of the two antibody concentrations and then experimentally confirm the result.

To this end, the signal yields and $\mathrm{ED}_{50}$ of Model $\mathrm{A}$ and $\mathrm{B}$, normalized to a conventional assay, are plotted against $\left[A b_{t 2}\right] /\left[A b_{t 1}\right]$ (Fig. 6) by using eqs. (8) and (10) divided by (9), respectively. Model B containing $A b_{2}$ with a lower binding constant $\left(K_{a 1} / K_{a 2}=48\right)$ produced a higher signal yield at an optimal value of $\left[A b_{t 2}\right] /\left[A b_{11}\right]$ (Fig. 6, upper panel). To the contrary, Model A with $A b_{2}\left(K_{a 1} / K_{a 2}=4.5\right)$ is better suitable for a more sensitive assay (Fig. 6, lower panel). The optimal values derived from these calculations are shown in Table I (without parentheses). 
Table I. Factors derived from the optimum calculated for $\left[A b_{t 1}\right] /\left[A b_{t 2}\right]$ (values without parenthesis) compared with the factors obtained under the applied experimental conditions (in parenthesis) for Models A and B.

\begin{tabular}{lcccc}
\hline \multicolumn{1}{c}{$K_{a 1} / K_{a 2}{ }^{\mathrm{a}}$} & {$\left[A b_{t 2}\right] /\left[A b_{t 1}\right]^{\mathrm{b}}$} & {$\left[\mathrm{Co}_{t}\right] /\left[A b_{t 1}\right]^{\mathrm{c}}$} & Sign. yield ratio $^{\mathrm{d}}$ & $\mathrm{ED}_{50}$ ratio $^{\mathrm{c}}$ \\
\hline 4.5 (Model A, BQ.1:E47) & $1.5(1.1)$ & $0.94(0.78)$ & $1.2(1.2)$ & $0.36(0.29)$ \\
48 (Model B, BQ.1:9G9 & $7.0(3.8)$ & $1.0(0.78)$ & $1.4(1.4)$ & $0.47(0.78)$ \\
\hline
\end{tabular}

${ }^{a} K_{a 1}$ was constant $\left(5.8 \times 10^{10} \mathrm{~L} / \mathrm{mol}\right)$, and $K_{a 2}$ was variable.

${ }^{\mathrm{b}}\left[A b_{t 1}\right]$ was constant $\left(2.4 \times 10^{-10} \mathrm{~mol} / \mathrm{L}\right)$, and $\left[A b_{t 2}\right]$ was variable; theoretical optimal values derived from Figure 6, upper panel.

${ }^{\mathrm{c}}$ Theoretical values were calculated by using eq. (6.4) with the values for $K_{a 1} / K_{a 2}$ and $\left[A b_{i 1}\right] /\left[A b_{i 2}\right]$ from the same row in Table $I$.

${ }^{d}$ Theoretical values were derived from Figure 6, upper panel, experimental values from Figure 7.

Theoretical values were derived from Figure 6, lower panel, experimental values from Figure 8 .

We hàd technical limitations in immobilizing the optimal amount of antibodies on the solid matrix. We were limited with the available volume and, consequently, with the amount of solid matrix in our system so that we could meet the requirements for optimal antibody ratios only for Model A but not for Model B. The experimentally obtained values shown in Table $\mathrm{I}$ in parentheses, therefore, differ for Model B from the calculated optimum (derived from Fig. 6).

\section{Conjugate Concentration}

Before we could perform dose-response curves with the dual-antibody assay, the only component remaining to
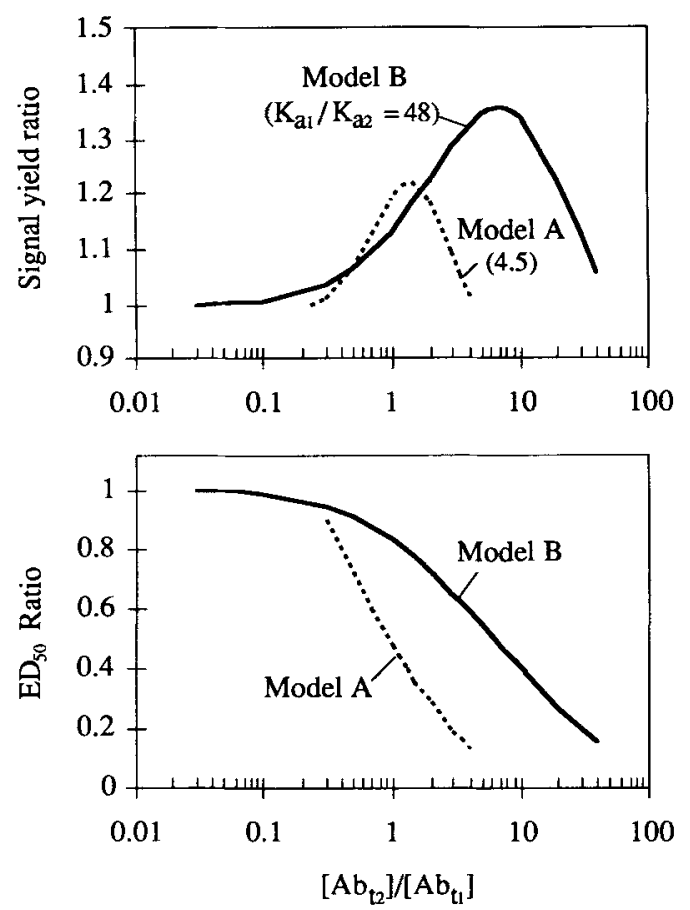

Figure 6. Theoretical signal yield ratio (eq. 8) and $E D_{50}$ ratio [eq. (10)/(9)] as a function of $\left[A b_{t 2}\right] /\left[A b_{t 1}\right]$ and the $K_{a 1} / K_{a 2}$ at constant $K_{a 1}\left[A b_{t 1}\right]=13.9$. At a given $K_{a 1} / K_{a 2}(4.5$ for Model $\mathrm{A}$ and 48 for Model B; see Table I for antibodies used), optimal $\left[A b_{t 2}\right] /\left[A b_{t 1}\right]$ was determined to obtain the maximal signal yield ratio. assess was the optimal concentration of the analyteenzyme conjugate. For the amount of antibodies that have been optimized as described earlier, the appropriate conjugate concentrations could be calculated from eq. (6.4). The calculated values for $\left[C o_{t}\right] /\left[A b_{t 1}\right]$, corresponding to the antibody concentrations that give the highest signal yield ratios, are shown in Table I (without parentheses). In our experiments, we used about $80 \%$ of the theoretically calculated concentration of progesterone-horseradish peroxidase conjugate because we wanted to avoid using standards with a high concentration to prevent potential precipitation of the steroid.

\section{Dose-Response Curves}

In a traditional competitive immunoassay, a sigmoidal dose-response curve is obtained where the signal is reversibly proportional to the dose of the analyte. For the dual-antibody system, we can construct two sigmoidal curves: one that is similar to the traditional assay (derived from binding at $A b_{1}$ ) and another one that is direct proportional to the analyte concentration (derived from binding at $A b_{2}$ ). These two curves are then combined by subtracting the signal at each concentration, to obtain a differential signal as reported elsewhere. ${ }^{15}$ All these operations can be easily performed by simple algorithms. This third dose-response curve, normalized to express the total concentration of bound analyte-enzyme conjugate as a function of known analyte added, is finally used for the quantification of samples.

Two such dose-response curves, Models A and B, are shown in Figure 7 and are compared with a curve obtained from a conventional assay. The total conjugate that contributes to signal generation ( $y$ axis) is expressed here as a function of analyte added. Depending on the selection of the two antibodies in the dualantibody system, the initial amount of conjugate bound and the displacement in the presence of different amounts of analyte do not parallel bound conjugate in a conventional assay.

We used the same amount of conjugate and $A b_{1}$ for all experiments shown in Figure 7. Therefore, the amount 


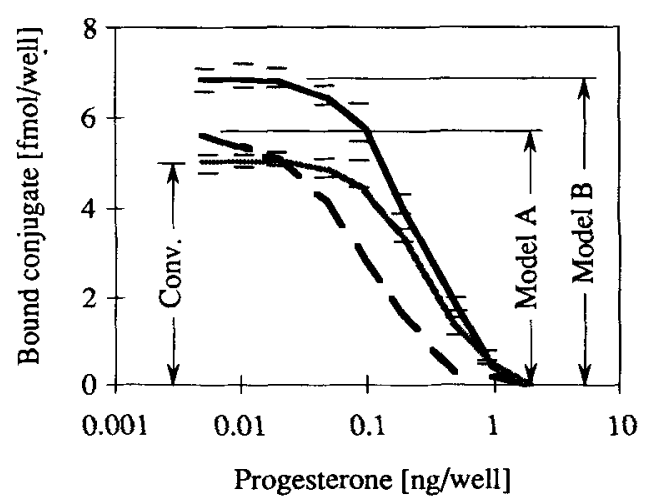

Figure 7. Bound conjugate in the dual-antibody assay (Models A and $B$ ) and a conventional competitive assay as a function of increasing analyte (progesterone) concentrations. The bound conjugate for the dual-antibody assays is expressed as a differential signal for normalized curves as described elsewhere. ${ }^{15}$

of bound conjugate in the absence of progesterone reflects directly the signal yield according to our definition (Definition of Terms). As expected from the theoretical calculations (Table I), the conventional assay has a lower signal yield (absence of analyte), although the curve crosses over that of Model A at increasing concentrations of progesterone at the expense of sensitivity (discussed later).

The experimental signal yield ratios of the models and the conventional assay coincided well with those theoretically obtained (Table I). Comparing the two models, the signal yield was greater with Model B. These results experimentally prove the first hypothesis, and support the theoretical prediction that the dualantibody system with a higher $K_{a 1} / K_{a 2}($ Model B) utilizes more efficiently the analyte-enzyme conjugate and generates a higher signal.

To compare the sensitivity at $50 \%$ displacement of analyte-enzyme conjugate $\left(E D_{50}\right)$, the three doseresponse curves in Figure 7 were transformed into a traditional format, i.e., a plot of the ratio of the signal in the presence of analyte $(B)$ over that in the absence of analyte $\left(B_{0}\right)$ vs. the native analyte concentration (Fig. 8). By comparing the $\mathrm{ED}_{50}$ of the three systems, Model A required about three times less analyte than the conventional assay (see $\mathrm{ED}_{50}$ ratio in Table I). The $\mathrm{ED}_{50}$ of Model $\mathrm{B}$ and the conventional assay differed only by $20 \%$ in favor of higher sensitivity for the dualantibody system. Under optimal conditions, up to about $50 \%$ higher sensitivity can be obtained for Model B (Table I), but we used in our experiment less than the optimal amount of enzyme antibody. The experimental results agreed well with the theoretical predictions that the dual-antibody model with a lower $K_{a 1} / K_{a 2}$ (Model A) can provide higher sensitivity (Table I). These experiments confirmed the second hypothesis.

It is noteworthy that the logit-log transformations ${ }^{12}$ of the sigmoidal dose-response curves (Fig. 8) showed that the slope of the curve from the single antibody assay was steeper than those from the two models of the dual-

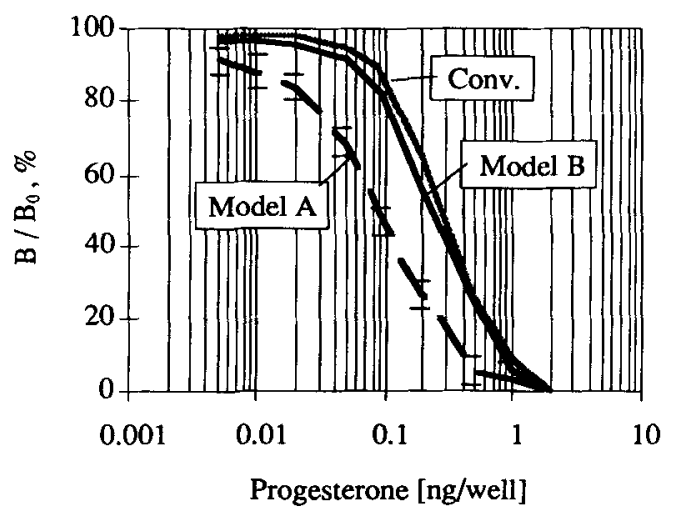

Figure 8. Comparison of sensitivities in dose-response curves in a traditional format by transforming the plot in Figure 7. Comparing the effective doses of progesterone to obtain $50 \%$ of $B / B_{0}\left(E_{50}\right)$, Model A increased sensitivity more than three times as compared to that of the conventional system. The sensitivity increase with Model A was greater than that with Model B.

antibody system. Therefore, the detectable range of the analyte using the dual-antibody method is not only lower, but is also wider.

\section{Limitations and Applications}

Provided we have an antibody, $A b_{1}$, with a given binding constant to the analyte, $K_{a 1}$, and an analyte-enzyme conjugate, $\mathrm{Co}$, which performance characteristics would we expect if we optimized two assays with these reagents, a conventional enzyme immunoassay and the dual-antibody assay?

In the dual-antibody system, the two dimensionless variables $K_{a 1}\left[A b_{t 1}\right]$ and $\left[C_{t}\right] /\left[A b_{t 1}\right]$ need to be carefully balanced to take advantage of the higher signal yield and sensitivity. This is different in a conventional assay where the only restriction is that a sufficient amount of conjugate is bound to $A b_{1}$ so that a reasonable signal can be obtained.

For the dual-antibody assay apply these limitations:

1. $K_{a 1}\left[A b_{t 1}\right]$. The basic concept is the shuttling of tracer from one compartment $\left(A b_{1}\right)$ to the other $\left(A b_{2}\right)$ in response to analyte concentration. For maximal shuttling, the amount of tracer bound at $A b_{1}$, which depends on $K_{a 1}\left[A b_{a 1}\right]$, has to be high in the absence of analyte so that a significant differential signal is obtained in the presence of analyte (see Figs. 2-5 for the performance comparisons). An optimal value for $K_{a 1}\left[A b_{t 1}\right]$, determined for the dual-antibody system, may be different from that for a single antibody assay. For example, in a solid-phase radioimmunoassay $^{17}$ with the same antibody (BQ.1) an optimal $\mathrm{ED}_{50}\left(4-5 \times 10^{-30} \mathrm{~mol} / \mathrm{L}\right.$ progesterone $)$ was obtained at $K_{a 1}\left[A b_{t 1}\right] \approx 1$. However, the enzyme-shuttle system at this condition is not superior to a conventional assay (Figs. 2-5).

2. $\left[\mathrm{Co}_{t}\right] /\left[A b_{t 1}\right]$. Another precondition for enzyme shuttling is the competition between the two antibodies 
for the capture of the signal generator. The degree of this competition is inversely proportional to the value of $\left[\mathrm{Co}_{t}\right] /\left[\mathrm{Ab_{t1 }}\right]$. If $\left[\mathrm{Co}_{t}\right] /\left[\mathrm{Ab} b_{t 1}\right]$ is higher than optimal, the performance of the dual-antibody system will approach that of a single antibody assay. The value of $\left[\mathrm{Co}_{t}\right] /\left[A b_{t 1}\right]$ in a single antibody assay is typically greater than unity (i.e., excess of tracer is used), ${ }^{19}$ while in the dual-antibody assay, it is smaller than or approximately equal to unity (see Table I), which is a precondition for maximum shuttling and, therefore, for maximal signal generation.

In conclusion, the enzyme-shuttle method is more effective if a relatively large amount of antibody to the analyte is used. This applies for the measurement of many analytes that are found in higher concentration ranges $\left(10^{-6} \mathrm{~mol} / \mathrm{L}\right)$. Likewise, the antibody concentration needs to be increased in the analytical system if enzymes with a comparatively low specific activity (i.e., turnover rates) are used. In these cases, a high value of $K_{a 1}\left[A b_{t 1}\right]$ is required, and [Cot can also be proportionally increased. Otherwise, the advantage of the dual-antibody method is not so much in replacing conventional enzyme immunoassays but rather in new applications. For example, we are now applying the principle to simple test strips for the quantitative determination of analytes in liquids. Furthermore, the dualantibody method lends itself for analyte detection in the reversible mode. This makes possible the continuous monitoring of analytes in medium streams. ${ }^{15}$

These investigations were supported by the U.S. Army Research Office (246655-LS) and a grant from the National Institute of Health (NICHHD, HD22316). We thank Drs. Richard H. Smith and Paul A. Craig for critical discussions.

\section{NOMENCLATURE}

$\left[A b_{x}\right] \quad$ concentration of antibody $x$ unoccupied by antigen $\left(M / L^{3}\right)$

$\left[A b_{x}: C o\right]_{y}$ concentration of binding complex of antibody $x$ with heterobifunctional conjugate at state $y\left(M / L^{3}\right)$

$\left[A b_{t x}\right]$ total concentration of antibody $x$ (unoccupied and occupied by the specific antigen) $\left(M / L^{3}\right)$

$\left[A b_{1}: P\right]$ concentration of binding complex of analyte antibody with native analyte $\left(M / L^{3}\right)$

$B$ concentration of conjugate bound to analyte antibody in the presence of analyte (single-antibody system) $\left(M / L^{3}\right)$

$B_{0} \quad$ concentration of conjugate bound to analyte antibody in the absence of analyte (single-antibody system) $\left(M / L^{3}\right)$

$\left[\mathrm{Co}_{l}\right]$ total concentration of conjugate, i.e., unbound and bound to antibodies $\left(M / L^{3}\right)$

[Co]y concentration of unbound conjugate at state $y\left(M / L^{3}\right)$

$[\mathrm{Co}]_{50}$ average concentration of unbound conjugate between initial and final states $\left(M / L^{3}\right)$

$D S_{y} \quad$ differential signal at state $y\left(M / L^{3}\right)$

$D S_{50} \quad$ average differential signal between initial and final states $\left(M / L^{3}\right)$

$E_{50} \quad$ estimated dose of analyte for $50 \%$ inhibition of bound conjugate $\left(M / L^{3}\right)$

$K$ square root of the ratio of two binding constants (dimensionless)
$K_{a p} \quad$ binding constant of analyte antibody to native analyte at equilibrium $\left(L^{3} / M\right)$

$K_{a x} \quad$ binding constant of antibody $x$ at equilibrium $\left(L^{3} / M\right)$

$[P]$ concentration of analyte (progesterone) $\left(M / L^{3}\right)$

$r$ ratio of practical signal yield to the maximum in single-antibody system (dimensionless)

\section{Greek letters}

$\alpha \quad$ square root of the ratio of two antibody concentrations (dimensionless)

$\begin{array}{ll}\text { Subscripts } & \\ 1 & \text { specific to analyte (progesterone) } \\ 2 & \text { specific to enzyme (horseradish peroxidase) } \\ i & \text { initial (ground) state without analyte } \\ f & \text { final state with excess analyte } \\ p & \text { native analyte (progesterone) } \\ t & \text { total concentration (bound and unbound) }\end{array}$

\section{References}

1. Bachas, L. G., Meyerhoff, M.E. 1986. Theoretical models for predicting the effect of bridging group recognition and conjugate substitution on hapten enzyme immunoassay doseresponse curves. Anal. Biochem. 156: 223-238.

2. Borth, R. 1980. Mass-action model for radioimmunoassays and other saturation assays with atypical performance characteristics. Math. Biosci. 51: 187-197.

3. Bos, E. S., van der Doelen, A. A., van Rooy, N., Schuurs, A. H. 1981. 3,3',5,5'-Tetramethylbenzidine as an Ames test negative chromogen for horse-radish peroxidase in enzyme-immunoassay. J. Immunoassay 2: 187-204.

4. Chard, T. 1987. An introduction to radioimmunoassay and related techniques. Elsevier, Amsterdam.

5. Daunert, S., Payne, B. R., Bachas, L. G. 1989. Pyruvate carboxylase as a model for oligosubstituted enzyme-ligand conjugates in homogeneous enzyme immunoassays. Anal. Chem. 61: $2160-2164$.

6. Fraser, C. G., Browning, M. C. 1986. A plea for abandonment of the term "highly sensitive" for thyrotropin assays. Clin. Chem. 32: 569-570.

7. Gosling, J. P. 1990. A decade of development in immunoassay methodology. Clin. Chem. 36: 1408-1427.

8. Jackson, T.M., Ekins, R. P. 1986. Theoretical limitations on immunoassay sensitivity. J. Immunol. Meth. 87: 13-20.

9. Laurence, D. J., Wilkinson, G. 1974. Model for competitive binding assays - the shape and location of the inhibition curve. Anal. Chem. 46: 1132-1135.

10. Matson, R.S., Little, M.C. 1988. Strategy for the immobilization of monoclonal antibodies on solid-phase supports. J. Chromatogr. 458: 67-77.

11. Nakamura, R.M., Voller, A., Bidwell, D. E. 1986. Enzyme immunoassays: Heterogeneous and homogeneous system. p 27.1-27.20 In: D. M. Weir (ed.), Handbook of experimental immunology, vol. 1. Blackwell, Oxford.

12. Rodbard, D., Lewald, J. E. 1970. Computer analysis of radioligand assay and radioimmunoassay data. Acta Enocrinol. 64 (suppl. 147): 79-103.

13. Sanderson, C. J., Wilson, D.V. 1971. A simple method for coupling proteins to insoluble polysaccharides. Immunology 20 : 1061-1065.

14. Scatchard, G. 1949. The attractions of proteins for small molecules and jons. Ann. N. Y. Acad. Sci. 51: 660-672.

15. Schramm, W., Paek, S. H. 1991. Continuous monitoring of analyte concentrations. Biosensors \& Bioelectronics, in press.

16. Schramm, W., Paek, S. H., Yang, T. 1990. Enzyme-analyte conjugates as signal generators for amperometric immunosensors: Immunochemical phenomena related to the detection of 
hapten molecules. p 153-170 In: R. P. Buck, W. E. Hatfield, M. Umana, and E.F. Bowden (eds.), Biosensor technology. Marcel Dekker, Inc., New York.

17. Schramm, W., Yang, T., Midgley, A. R. 1987. Monoclonal antibodies used in solid-phase and liquid-phase assays, as examplified by progesterone assay. Clin. Chem. 33: 1331-1337.
18. Steward, M.W., Lew, A. M. 1985. The importance of antibody affinity in the performance of immunoassays for antibody. J. Immunol. Meth. 78: 173-190.

19. Zettner, A. 1973. Principles of competitive binding assays (saturation analyses). I. Equilibrium techniques. Clin. Chem. 19: $699-705$. 